\title{
Zur Frage über die Ansteckung an Tuberkulose jüdischer Kinder während der Beschneidung ${ }^{1}$ ).
}

\author{
Von \\ Dr. med. I. M. Arluck und Dr. med. I. J. Winocouroff. \\ Mit 1 Tafel.
}

Fälle von Ansteckung an Tuberkulose jüdischer Kinder während des Beschneidungsritus werden heutzutage in Städten so gut wie nie angetroffen. Seitdem die Kenntnisse der Hygiene mehr und mehr das Bewusstsein der Volksschichten durchdringen, seitdem das Volk zur Überzeugung kam, dass der Ritus durch die Art der Blutstillung keine Einbusse leidet, ist die Ansteckung in den Städten fast unmöglich geworden. Aber in der abgelegenen Provinz, wo Dunkelund Unwissenheit herrschen, kommen solche Fälle nicht selten vor. Wenn wir darüber wenig wissen, so geschieht es nur deshalb, weil diese Fälle in die medizinische Presse nicht gelangen. Hauptsächlich dadurch ist zu erklären, warum die Literatur dieser Frage nicht so reich ist.

So beschrieb Lindemann (im Jahre 1893) zwei Fälle von Ansteckung an Tuberkulose während der Beschneidung, wobei der Operateur, der das Blut ausgesogen hat, tuberkulös war. Bei beiden Kindern wurden tuberkulöse Ulzera am Penis und käsige Degeneration der Inguinaldrüsen beobachtet; ein Fall ist zum Exitus gekommen, im anderen trat Genesung nach geräumiger Zeit ein. Der Operateur starb nach zwei Monaten nach der oben beschriebenen „Operation".

Lehmann beobachtete bei 10 Kindern nach 10 Tagen nach der Beschneidung tuberkulöse Geschwüre, nach drei Wochen Schwel-

1) Vortrag gehalten in der Gesellschaft Odessaer Ärzte im Jahre 1910 von Dr. med. I. M. Arluck. 
lung und Abszess der Inguinaldrüsen. Operateur war schwindsüchtig. Elsenberg beschrieb 4 Fälle tuberkulöser Affektion des Penis, die von allgemeiner Tuberkulose gefolgt war. In den Familien aller dieser Kinder wurde keine Tuberkulose konstatiert. Im Sputum des Operateurs wurden $\mathrm{Kochsche} \mathrm{Bazillen} \mathrm{gefunden.} \mathrm{Ähnliches}$ wurde von anderen Autoren beobachtet ( $H$ of mokl, v. Bergmann, Mayer, Eve, Löwenstein, Gescheit u. a.). Alle diese Arbeiten gehören zu den 80 er und zum Anfang der 90 er Jahre. Nebenbei sei bemerkt, dass in diesen Arbeiten von einer vorgenommenen Sektion nichts erwähnt ist. In der russischen Literatur ist diese Frage sehr mangelhaft beleuchtet. Einzige Fälle sind von Kolzow, Pasternazky, Tschainsky u. a. beschrieben. Kolzows Arbeit, die im Jahre 1891 in der Zeitschrift der Russischer Gesellschaft für Volksgesundheitsfürsorge erschienen, ist besonders hervorzuheben. Aus den 7 von $\mathrm{Kolzow}$ beschriebenen Fällen ist einer genesen, zwei starben, zwei nur unvollkommen g'enesen, zwei sind aus der Beobachtung des Autors verschwunden.

In allen diesen Fällen, einen ausgenommen, konnte in der Familie keine Tuberkulose konstatiert werden. Kolzows Arbeit gewann eine grosse soziale Bedeutung. Es ist dieser Arbeit zu verdanken, dass eine Kommission im Jahre 1891 in Petersburg aus den Vertretern der Medizin, der Gesellschaft, Spezialisten in jüdischen rituellen Angelegenheiten einberufen worden war, die beschlossen hat: dass der Beschneidungsritus zu reformieren ist, dass er streng hygienisch durchgeführt werden muss, um so mehr, da das $\mathrm{Ab}$ saugen des Blutes mit dem Munde und die Abreissung der Lamina interna mit den Fingern überhaupt kein unbedingt notwendiges Attribut des Rituals zu sein braucht.

Wenu wir nun nach Angabe der betreffenden Literatur unseren Fall doch ausführlich besprechen möchten, so geschieht es erstens deshalb, weil er eine soziale Bedeutung gewinnen kann und zweitens darum, weil eine Obduktion des verstorbenen Säuglings sehr wichtige Aufschlüsse über die Frage der Verbreitung der tuberkulösen Infektion aus dem Urogenitalsystem nach dem ganzen Organismus geliefert hat.

Jetzt zu unserem Fall:

Kind von $5^{1 / 2}$ Monaten, stammt von gesunden Eltern (Vater 38 Jahre, Mutter 32 Jahre alt). Die letzteren wurden von uns untersucht und gesund gefunden. In der Familie fünf Kinder - alle gesund. Zwei Aborte: einer im 6. Monat, ein zweiter im 8. Ein Kind starb am 8. Tag nach der Geburt. Unser Patient ist zur rechten Zeit geboren; die Geburt verlief normal; weder Ausschlag noch sonst 
irgendwelche Krankheiten der Neugeborenen. Das Kind wurde die ganze Zei vo von der Mutter gestillt; ausser leichten dyspeptischen Erscheinungen keine Krankheiten. Am 8. Tag nach der Geburt wurde die Beschneidung nach dem alten Usus vorgenommen, und zwar, einer der eingeladenen Gäste (der nach Aussage der Eltern des Kindes hüstelte) sog das Blut aus. Nach zwei Wochen bemerkte die Mutter am Penis des Kindes Rötung und Schwellung, die zwei Wochen lang anhielten; nachher erschienen kleine Ulzerationen, die Eiter absonderten; gleichzeitig fingen die Leistendrüsen an $\mathrm{zu}$ schwellen. Allmählich schritt die Krankheit fort und das Kind wurde zu uns, in die Kinderabteilung des jüdischen Spitals zu Odessa, aus einem unweit von Odessa sich befindlichen Gute in folgendem Zustande eingeliefert:

St. praes. Kind von sehr schwachem Ernährungszustand; Haut und Schleimhäute anämisch. Zervikal- und Axillardrüssen ein wenig vergrössert. Zunge stark belegt (Soor). Rachen rein. Lungen- und Perkussionston normal. Bei der Auskultation wird rauhes Atmen konstatiert. Herz normal. Puls 116 in der Minute, regelmässig von schwacher Füllung. Leber und Milz nicht vergrössert. Urin normal. Stuhl 3-4 mal täglich, dyspeptisch. Das Nervensystem zeigt keine Abweichungen von der Norm. Was den Lokalbefund anbetrifft, so stellt ex sich folgendermassen dar: Der ganze Penisschaft bis zur Anheftungsstelle am. Skrotum ist von der Haut entblösst; Glans penis ist in der unteren Abteilung gespalten, so dass ein Orificium externum nicht mehr existiert. Der ganze Penisschaft stellt eine grosse ulzeröse Oberfläche, die sich in die Tiefe erstreckt, dar. Beim Zurückschlagen des Skrotum wird eine ulzeröse Oberfläche mit speckig belegtem Boden und unterminierten verdickten Rändern konstatiert. Inguinaldrüsen stark vergrössert, von verschiedener Konsistenz, gesondert, palpabel.

Die klinische Diagnose des Falles war nicht schwer. Das Aussehen des Geschwürs und der Charakter der Inguinaldrüsen sprach für Tuberkulose. Wir beschlossen aber durch andere Untersuchungsmethoden unsere Diagnose zu ergänzen und nachzuprüfen. $\mathrm{Zu}$ diesem Zweck wurde die Pirquetsche und die Wassermannsche Reaktion vorgenommen. Die letztere wurde von Dr. Brodsky, dem wir auch hier unseren Dank aussprechen, ausgeführt. Die beiden Reaktionen fielen negativ aus. Das Fehlen der Pirquetschen Reaktion erklärten wir uns durch den weit fortgeschrittenen tuberkulösen Prozess. Aus dem Inhalt der ulzerösen Fläche wurden einige Präparate angefertigt und nur in einem derselben ein einziges tuberkulöses Stäbchen gefunden. Unsere klinische Diagnose lautete also: 
Tuberkulose des Penis. Das Kind befand sich drei Tage auf der Kinderabteilung und starb am 4. Tage unter Erscheinungen von Herzschwäche. In diesen drei Tagen schwankte die Temperatur zwischen $38^{\circ}$ und $37,6^{\circ} \mathrm{C}$; in den Lungen wurden nur unbedeutende katarrhalische Erscheinungen beobachtet; der Puls war während der ganzen Zeit von schwacher Füllung, regelmässig, ca. 120-130.

Die von Priv.-Doz. der Neurussischen Universität (z. Zt. Prof. der Warschauer Universität) Dr. Poscharisky vorgenommene Obduktion hat folgendes ergeben: Leiche eines stark abgemagerten Kindes; Zwerchfell rechts am 5 ., links am 6 . Interkostalraum. Beide Lungen am Durchschnitt für Luft durchgängig; hier und da im Lungengewebe disseminierte, durchsichtige, nicht zahlreiche, miliare, graue Knötchen. Drüsen am Lungenhilus vergrössert, hellrosa gefärbt; in einigen derselben gelbe Stellen; Halsdrüsen mässig gross, von grauer Farbe mit deutlich erkennbaren, trockenen, gelben Flecken. Schleimhaut der Trachea, des Ösophagus und des Kehlkopfes ohne deutliche Veränderungen. Leber mässig gross, leimfarben, morsch. Milz gross, Pulpa von hellroter Farbe, am oberen Rand der Pulpa zwei trockene gelbe Knötchen, jedes von Hanfkorngrösse. Nieren ohne besondere Veränderungen. Magen ist mit Milchkoagulis gefüllt, Schleimhaut desselben dünn. Im Dünn- und Dickdarm zerstreut etwa sieben Ulzera von unregelmässiger Form mit umgekrempelten festen Rändern. Mesenterialdrüsen mässig gross; in den meisten sind deutlich grosse, trockene, graugelbe Stellen zu seher. Die Lymphdrüsen im kleinen Becken, ebenfalls an den Rändern des Hüftbeins unter dem Peritoneum, Inguinaldrüsen, die grosse ovoide Körper von $3 \mathrm{~cm}$ Länge darstellen - alle weisen auf dem Durchschnitt trockene graugelbe Massen auf. Die Haut am Penis fehlt, kavernöse Körper sind entblösst und haben das Aussehen von roten Körpern; Glans penis von unregelmässiger Form. Die anatomische Diagnose: Ulcera tuberculosa tractus intestinalis; Ulcus tuberculosum cutis penis; Degeneratio caseosa glandularum lymphaticarum pelvis minoris, mesenterii et inguinalium. Tuberculosis miliaris pulmonum, lienis, glandularum lymphaticarum colli et bronchialium. (Auf dem nach der Sektion von den Mesenterialdrüsen angefertigten Abstrichpräparat waren tuberkulöse Stäbchen gut zu sehen.)

Bevor wir zur Analyse des Sektionsmaterials, das nur ein theoretisches Interesse beansprucht, übergehen, möchten wir auf die sozial-medizinische Seite dieser Frage die Aufmerksamkeit lenken. Die Tuberkulose bei Kindern und besonders im Säuglingsalter hat ihre besonderen charakteristischen Merkmale, die von den anatomisch- 
physiologischen Eigentümlichkeiten des kindlichen Organismus, sowie von einer Reihe sozialer Bedingungen abhängig ist. Ein Blick auf die Tabellen (Pirquet, Ha mburger u. a.) über die Erkrankung und Sterblichkeit an Tuberkulose genügt, um sich zu überzeugen, wie selten eigentlich die Tuberkulose bei Kindern bis zu einem Jahre, besonders nach den Monaten gerechnet (vom 1. bis zum 12. Monat), und wie andererseits gross die Mortalität infolge der Schutzlosigkeit dieses zarten Alters ist. Ziehen wir diese auf einer grossen Anzahl von statistischen Zahlen beruhenden Angaben in Erwägung, so müssen wir sagen, dass die Beschneidung nach dem alten Modus, der noch jetzt üblich ist, nur künstlich die Sterblichkeit der Kinder an Tuberkulose im frühen Säuglingsalter erhöhen kann. Deshalb können alle Bestimmungen betreffend der Vervollkommnung dieses Ritus im Sinne der strengen Beachtung der Hygiene eine grosse soziale Bedeutung gewinnen.

Das Interesse dieses Falles wird aber nicht nur durch seine soziale Bedeutung erschöpft; er beansprucht unsere Aufmerksamkeit durch die Wege, die sich die tuberkulöse Infektion bei der Verbreitung im Organismus bedient hat. Aus dem Sektionsprotokoll ersehen wir: zunächst eine Affektion des lymphatischen Systems verschiedenen Grades von den Inguinal- bis zu den Zervikaldrüsen hinauf; nebstdem unbedeutende Veränderungen in den Lungen und in der Milz, bei vorhandenen Geschwüren im Darm (sechs Geschwüre im Dünndarm, ein Ulkus im Duodenum). Wenn wir die geschilderten Verhältnisse überblicken, so drängt sich die Frage auf, wie könnte es vorkommen, dass die Sektion ein so ungewöhnliches Bild geliefert hat.

En g e l sagt in seiner Monographie: „Die Pathologie der Kindertuberkulose", dass im Säuglingsalter die am meisten typische pathologisch-anatomische Form der Kindertuberkulose entweder in der Form dei tuberkulösen Affektion nur des Lymphsystems allein (allgemeine Lymphdrüsentuberkulose) oder zusammen mit der submiliaren Aussaat in den Organen vorkommt. Der dritten Form (Lymphdrüsentuberkulose und Miliartuberkulose) begegnet man ziemlich selten.

Gehen wir von diesen typischen Formen zu den Sektionsprotokollen der im Säuglingsalter an Tuberkulose gestorbenen Kinder über, so ersehen wir, dass tuberkulöse Geschwüre im Darm in denjenigen Fällen angetroffen werden, in welchen die Tuberkulose in anderen Organen stark ausgeprägt ist, besonders in den Lungen und in den peribronchialen Drüsen, d. h., dass alle diese Geschwüre in der Mehrzahl der Fälle sekundärer Natur sind. Heubner sagt 
im Kapitel über Darmtuberkulose bei Kindern, dass er tuberkulöse Darmgeschwüre bei einem sechsmonatlichen Kinde (sekundäre Darmtuberkulose) und nebstdem käsige Degeneration in der Lunge gesehen habe. In einem anderen Falle sah er bei einem Säugling einen Ulkus im Darm (primäre Tuberkulose?).

In unserem Falle begegnen wir nur in zwei Organen, in den Lungen und in der Milz, hier und da einer geringen Zahl miliarer und grauer Knötchen. Wie ist nun die Herkunft tuberkulöser Geschwüre im Darm zu erklären?

Wenr die tuberkulöse Infektion während der Beschneidung gleichzeitig durch die Lymphe und durch das Blut hineingelangt wäre, so hätten zusammen mit der Affektion des Lymphsystems auch tuberkulöse Infektionen in den anderen Organen, und hauptsächlich in den Lungen, wie es aus Versuchen an Tieren bei Ansteckung derselben durch das Gefässsystem festgestellt ist, vorhanden sein müssen. Sollte sogar $\mathrm{Saw}$ adas Theorie richtig sein, dass Kinder in den ersten Jahren ihrer Lebzeit infolge der ungenügenden Entwickelung der Lymphknötchen in ihren Lungen selten an miliarer Lungentuberkulose erkranken, so muss man auch nicht vergessen, dass im frühesten Säuglingsalter der Darm das tuherkulöse Gift nicht fixiert.

Wir sehen also, dass bei sehr unbedeutenden Erscheinungen in den Lungen und in der Milz und beim Fehlen derselben in anderen Organen die Entstehung der Darmgeschwïre auf hämatogenem Wege nicht zu erklären ist. Wir muissen deshalb zugeben, dass die tuberkulöse Infektion während des Beschneidungsritus durch die Lymphe eingedrungen ist. Soll die Entstehung der Darmgeschwüre auf dem Lymphwege, d. h. durch das Eindringen des tuberkulösen Giftes aus den Mesenterialdrüsen in den Darm auf retrogradem Wege, d. h. in der dem Lymphstrom entgegengesetzten Richtung zu erklären sein? Der retrograden lymphogenen Infektion begegnet man nicht oft, eine solche Erscheinung aber ist in der experimentellen Pathologie, wie es weiter aus den Versuchen Cornets an Meerschweinchen ersichtlich ist, bekannt.

Wenn wir noch hinzufügen, dass zur Ansteckung des Darms an Tuberkulose ein grosses Quantum des Giftes, welches dorthin aus den Mesenterialdrüsen, zum grössten Teil des Dünndarmmesenteriums, transportiert werden muss, so wird es rerständlich, warum wir in diesem Falle eine so starke Affektion des Darms haben (sechs Geschwüre im Dünndarm und ein Dickdarmulkus). Was Lungen und Milz anbetrifft, so kann man sich vorstellen, dass auch dort die Infektion auf retrogradem Wege gelang, obwohl wir die Infektion 
dieser Organe auf hämatogenem Wege nicht ausschliessen können, was aber zu der Zeit, nachdem das tuberkulöse Gift den grössten Teil des Weges lymphogen passiert hatte, geschehen müsste. Es fragt sich nun, ob wir zur Analyse unseres Sektionsmaterials die zahlreichen Experimente und Schlussfolgerungen aus der experimentellen Pathologie hinzuziehen und eine volle Analogie durchführen dürfen. Wenn in anderen Fällen eine solche Analogie in vollen $\mathrm{H}_{\perp}$ Umange infolge einer ganzen Reihe von Bedingungen, wie das Alter des Patienten, der Unbekanntheit der primären Affektion nicht durchführbar ist, so können wir in unserem Falle sagen, da das Kind von völlig gesunden Eltern stammte, von der Mutter gestillt und nicht von Tuberkulösen umgeben war, dass während der Beschneidung an ihm ein wirkliches, leider trauriges Experiment vollzogen wurde.

Wenden wir uns jetzt zur experimentellen Pathologie. Aus Experimenten an 4000 Meerschweinchen zieht Cornet folgende Schlüsse :

1. Die tuberkulöse Infektion breitet sich ohne Sprünge, Schritt für Schritt im Organismus aus.

Die zahlreichen Untersuchungen von Cornet wurden von W lem insky an 1000 Schweinchen bestätigt.

2. Eine besondere Affinität einzelner Organe zum tuberkulösen Gift existiert laut den Experimenten nicht. Alles hängt von der Invasionsstelle der Infektion, der Zahl und Virulenz der tuberkulösen Bazillen ab. So wird z. B. die Lunge zuerst affiziert, wenn die Infektion direkt in die Luftwege gelangt und am spätesten, wenn die Invasionsstelle weit von ihr entfernt liegt.

3. Die tuberkulöse Infektion schreitet hauptsächlich mit dem Lymphstrome (zentripetal) fort, selten senkrecht zum Lymphstrom, am seltensten in einer dem Lymphstrom entgegengesetzten Richtung.

Ähnliches beobachten wir auch in unserem Falle. Die Infektion ergriff alle Drüsen von den Leisten- bis zu den Halsdrüsen hinauf. Die peribronchialen Drüsen, die unter gewöhnlichen Bedingungen öfter und stärker als andere Drüsen beim Säugling affiziert werden, in unserem Falle, unter Bedingungen eines Experiments, da weit vom Infektionsort entfernt, am wenigsten betroffen gefunden wurden; der Darm, der im Säuglingsalter selten an Tuberkulose erkrankt, war in unserem Falle infolge der Nähe vom Ansteckungsort und der Menge und Virulenz des von den Mesenterialdrüsen produzierten Giftes stark infiziert. In diesem Falle schritt die Infektion meist mit der. Lymphstrom zusammen vor (von den Leisten- bis zu den peribronchialen Drüsen), weniger wurde der Weg von den Drüsen 
zu den Organen eingeschlagen, und viel weniger breitete sich die Infektion in der dem Lymphstrom entgegengesetzten Richtung aus (von den peribronchialen bis zu den Leistendrüsen).

4. Bei Infektion durch Blutgefässe werden Tuberkeln in allen Brust- und Baucheingeweiden im gleichen Entwickelungsstadium angetroffen; in den Lungen sind sie etwas zahlreicher, da bei hämatogener Infektion die Lungen den ersten Filter darstellen. In unserem Falle konstatieren wir dies nicht, deshalb schliessen wir den hämatogenen Weg aus.

Gehen wir jetzt von den allgemeinen von Cornet und Wlemansky aus Experimenten an 5000 Meerschweinchen gewonnenen Resultaten zu den speziellen Untersuchungen Cornets über:

1. Wenn eine Zehe an Tuberkulose angesteckt wird, so konstatieren wir nach einiger Zeit eine käsige Degeneration der Kniekehlen-, Leisten- und retroperitonealen Drüsen; Tuberkulose der Leber und der Milz; wenn das Tier noch später getötet wird, so gesellt sich Tuberkulose der Lungen und der peribronchialen Drüsen hinzu; wenn die Infektion von oben eindringt, z. B. von der Nase, so bekommen wir ein ganz umgekehrtes Bild.

2. Wenn in den Penis tuberkulöses Sputum oder eine Reinkultur eingerieben wird, so entstehen bei unbedeutender Iäsion des Penis umfangreiche Ulzera; nach 2-3 Wochen schwellen und degenerieren die Leistendrüsen; später erkranken auch die retroperitonealen Drüsen; nach den anderen 2-3 Wochen werden Leber und Milz affiziert, und endlich nach 2 Monaten werden Tuberkeln in den Lungen konstatiert.

Cornets Lehre von dem lymphogenen Wege bei der Ausbreitung des tuberkulösen Giftes im Organismus und der Möglich. keit der retrograden Infektion wurde durch Untersuchungen von von Tendeloo, Sappey, Küttner über die Anatomie des Lymphsystems bestätigt.

Diese Forscher haben auf eine Reihe von Anastomosen hingewiesen, die zwischen den Lymphsystemen der Brust- und Bauchhöhle existieren. Hiermit ist es gelungen, eine Reihe der bis dahin unerklärlich gebliebenen Erscheinungen richtig zu deuten. Wir sehen also, dass nur das reiche Gebiet der experimentellen Pathologie uns in den Stand gesetzt hat, das auf den ersten Blick unklar erschienene Bild der Sektion zu erklären. 


\section{Literatur.}

1. Cornet, G. Prof., Die 'Tuberkulose, 1907.

2. Engel, Dr., Die Pathologie der Kindertaberkulose, Beihefte zur Medizinischen Klinik 1909.

3. Kejge, Saw oda Dr., Zur Kenntnis der hämatogenen Miliartuberkulose der Lungen. Deutsches Archiv für klinische Medizin 1903, 76. Bd.

4. He u bner, O. Prof., Lehrbuch der Kinderheilkunde 1906, 11. Bd.

5. Etlinger, Dr., Die Tuberkulose im Säuglingsalter 1908. Klinische Monographie, Ausgabe der Zeitschrift der „Praktischen Medizin“.

6. Kolzow, Dr., Zur Frage über die Ansteckung jüdischer Kinder während der Beschneidung an Tuberkulose. Zeitschrift der Russischen Gesellschaft der Volksgesundheitsfürsorge 1891. 


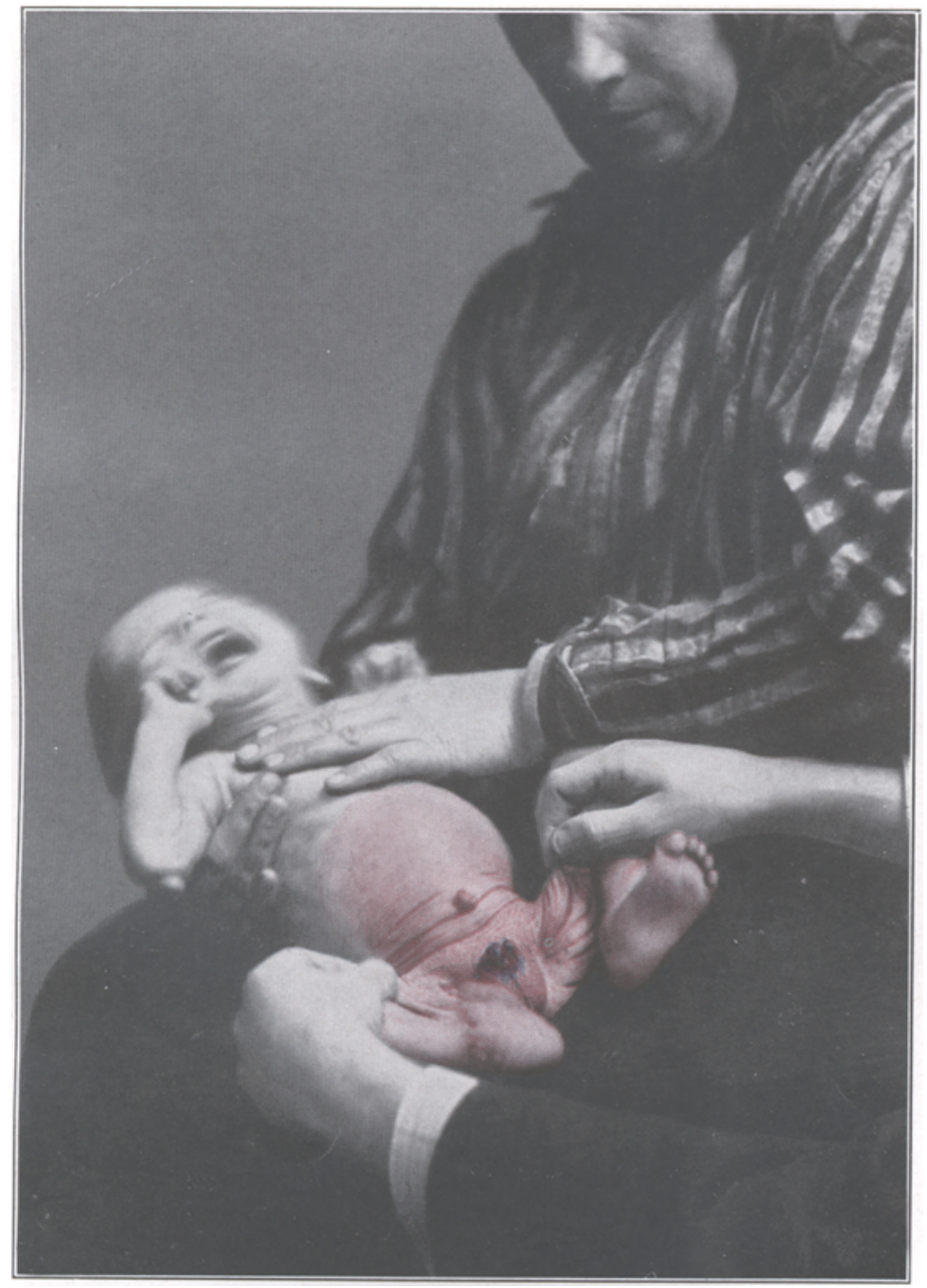

Arluck und Winocouroff, Zur Frage über die Ansteckung an Tuberkulose jüdischer Kinder während der Beschneidung. 